\title{
Mineração
}

\section{Avaliação de capacidade de um depósito típico de níquel laterítico aproveitado através de rota de lixiviação ácida sob pressão}

\section{(Capacity assessment of a typical PAL lateritic nickel deposit)}

\author{
Ruy Lacourt Rodrigues \\ Engenheiro de Minas, Universidade Federal de Ouro Preto \\ E-mail:ruy.lacourt@vale.com \\ Valdir Costa e Silva \\ Professor Associado, Universidade Federal de Ouro Preto \\ E-mail:valdir@demin.ufop.br
}

\section{Resumo}

Avaliou-se a capacidade de produção de uma operação de níquel laterítico, aproveitado através de rota de processo de lixiviação ácida sob pressão, para escalas de produção entre 45000 t/ano Ni e 300000 t/ano Ni, usando parâmetros da indústria.

A análise econômica foi feita utilizando a metodologia de fluxos de caixa descontados e análise de sensibilidade. Os indicadores de desempenho foram interpretados no contexto de avaliação de empreendimentos em mineração, o que permitiu delinear cenários para a recomendação de escala.

A escala recomendável é de 120000 t/ano Ni, que representa a melhor solução de compromisso tendo em vista a rentabilidade do empreendimento, a maturação da tecnologia e o tamanho do mercado.

Palavras-chave: Avaliação econômica em mineração, definição de capacidade, níquel laterítico, lixiviação ácida sob (alta) pressão (PAL, HPAL, HiPAL).

\section{Abstract}

The economic evaluation of production capacity for a lateritic nickel operation using pressure acid leach was performed for capacities from 45000 t/year Ni to 300000 t/year Ni using industry parameters.

The economic analysis was performed using discounted cash flow and sensitivity analyses. Performance indicators were interpreted in a mine valuation context, allowing to establish scenarios for capacity recommendation.

The recommended capacity is 120000 t/ano $\mathrm{Ni}$, which represents the best compromise considering profitability, the maturation of the technology and market size.

Keywords: Mining valuation, capacity definition, lateritic nickel, (high) pressure acid leaching (PAL, HPAL, HiPAL). 
Avaliação de capacidade de um depósito típico de níquel laterítico aproveitado através de rota...

\section{Introdução}

As lateritas de níquel, em especial aquelas em que o aproveitamento é feito através de rotas de lixiviação ácida sob pressão, são a maior fonte potencial para o crescimento da oferta do metal.

Os projetos que vêm sendo desenvolvidos através dessa linha tecnológica são de grande porte, a fim de que sejam capturadas economias de escala e diluídos os gastos com utilidades e infra-estrutura.

A definição da capacidade desses empreendimentos é complexa, pois envolve uma tecnologia que ainda está em amadurecimento, grande capital investido e operações com baixo teor de alimentação.

\section{Metodologia e premissas}

Considerando a qualidade, tamanho e locação de cada depósito mineral, não há procedimentos bem estabelecidos para a definição de capacidade de empreendimentos em mineração. A escolha da escala de produção é, em geral, delineada através de tentativa e erro a partir dos parâmetros para a definição de capacidade, ponderada pela análise do cenário estratégico que afeta cada projeto.

Uma revisão sobre o aproveitamento de níquel laterítico através das várias rotas de processo pode ser encontrada em Dalvi, Bacon e Osborne (2004), onde são feitas referências à capacidade mínima para projetos de aproveitamento de níquel laterítico por rotas de lixiviação ácida sob pressão para as condições de mercado da época.

Um depósito-tipo de tamanho médio foi definido a partir dos dados dos projetos em desenvolvimento e implementação e estabeleceu-se o plano de aproveitamento para várias escalas de produção a partir de parâmetros da indústria (Rodrigues, 2007).

Em seguida, desenvolveu-se a análise econômica do depósito para várias escalas de produção, utilizando a metodologia de fluxos de caixa descontados, o que permitiu estabelecer cenários que levam à recomendação de escala.

As premissas e fontes de dados principais para a análise econômica são mostradas na Tabela 1.
A análise de sensibilidade foi feita através da técnica de Monte Carlo, os parâmetros variáveis são, também, mostrados na Tabela 1.

\section{Considerações sobre as escalas de produçãoestudadas}

Foram feitos estudos para cinco escalas de produção diferentes (em 1000 t/ano Ni): 45, 60, 120, 200 e 300.

A despeito de grande variação quanto ao tamanho dos recursos, o limite superior de capacidade para os projetos em estudos e implementação é de 60000 t/ano Ni, conforme mostra a Figura 1.

A implementação do empreendimento em escalas muito grandes implica investimentos muito altos e o fornecimento de uma fatia de mercado muito expressiva a partir de uma única operação, dependente de tecnologia ainda em maturação, aumentando os riscos e limitando a escala dos empreendimentos.

Portanto as simulações a escalas muito elevadas consistem em um exercício que se justifica para se estabelecer uma amplitude de escala suficiente, a fim de se definir a capacidade recomendável.

Tabela 1 - Premissas e fontes de dados.

\begin{tabular}{|c|c|c|c|c|}
\hline \multirow{2}{*}{\multicolumn{2}{|c|}{ Parâmetro (unidade) }} & \multirow{2}{*}{ Fonte } & \multicolumn{2}{|c|}{ Valor / Cenário } \\
\hline & & & Caso base & Análise de sensibilidade \\
\hline \multicolumn{2}{|c|}{ Taxa de desconto } & & $12 \%$ & $8 \%$ a $20 \%$ \\
\hline \multirow{2}{*}{$\begin{array}{l}\text { Preços } \\
\text { (USD/lb) }\end{array}$} & $\mathrm{Ni}$ & \multirow{2}{*}{$\begin{array}{l}\text { Adaptados de Neudorf e } \\
\text { Huggins (2004) }\end{array}$} & 5,5 & 4,5 a 8,0 \\
\hline & Co & & 10 & 8 a 15 \\
\hline \multicolumn{2}{|c|}{$\begin{array}{l}\text { Investimentos iniciais } \\
\text { (USD/lba) }\end{array}$} & $\begin{array}{l}\text { Adaptados de Dolan e } \\
\text { Nendick (2004) }\end{array}$ & 20 & 16 a 25 \\
\hline \multicolumn{2}{|c|}{$\begin{array}{l}\text { Custos operacionais } \\
\text { (USD/lb) }\end{array}$} & $\begin{array}{c}\text { Hand (2006), ajustes } \\
\text { conforme Johnston (2006) }\end{array}$ & 2,0 & 1,5 a 3,0 \\
\hline \multicolumn{2}{|c|}{ Taxação } & DNPM (2000) & \multicolumn{2}{|c|}{ Toda a produção para exportação } \\
\hline \multicolumn{2}{|c|}{ Financiamento } & Barbosa (2007) & \multicolumn{2}{|c|}{$\begin{array}{c}40 \% \text { dos investimentos a juros de } 6,5 \% \text { a.a. com } 3 \\
\text { anos de carência e } 5 \text { anos para amortização do } \\
\text { principal }\end{array}$} \\
\hline
\end{tabular}


Ruy Lacourt Rodrigues et al.

\section{Resultados e discussão}

A Figura 2 mostra as relações entre o valor presente líquido e os investimentos iniciais e a escala de produção.

A análise a partir do valor presente líquido indica como recomendável a escala de 200000 t/ano Ni. Entretanto os investimentos são muito altos e a fatia de mercado fornecida é, também, muito grande.

Os investimentos iniciais para as escalas até 60000 t/ano $\mathrm{Ni}$ ou 120000 t/ano Ni, embora elevados no contexto da indústria de mineração, têm suporte na base de dados da indústria (Rodrigues, 2007, op. cit.); a partir daí, os valores são muito elevados.

A análise através da eficiência do uso do capital - relação entre o valor presente líquido do empreendimento e o valor presente dos investimentos - e da taxa interna de retorno, mostrada na Figura 3, oferece maiores subsídios para a decisão.
A eficiência de capital é um parâmetro mais adequado que o valor presente líquido para a escolha da escala recomendável, na medida em que esta-

belece a relação entre valor recuperado e os investimentos. Considerando esse parâmetro, a escala recomendável é de 120000 t/ano Ni.

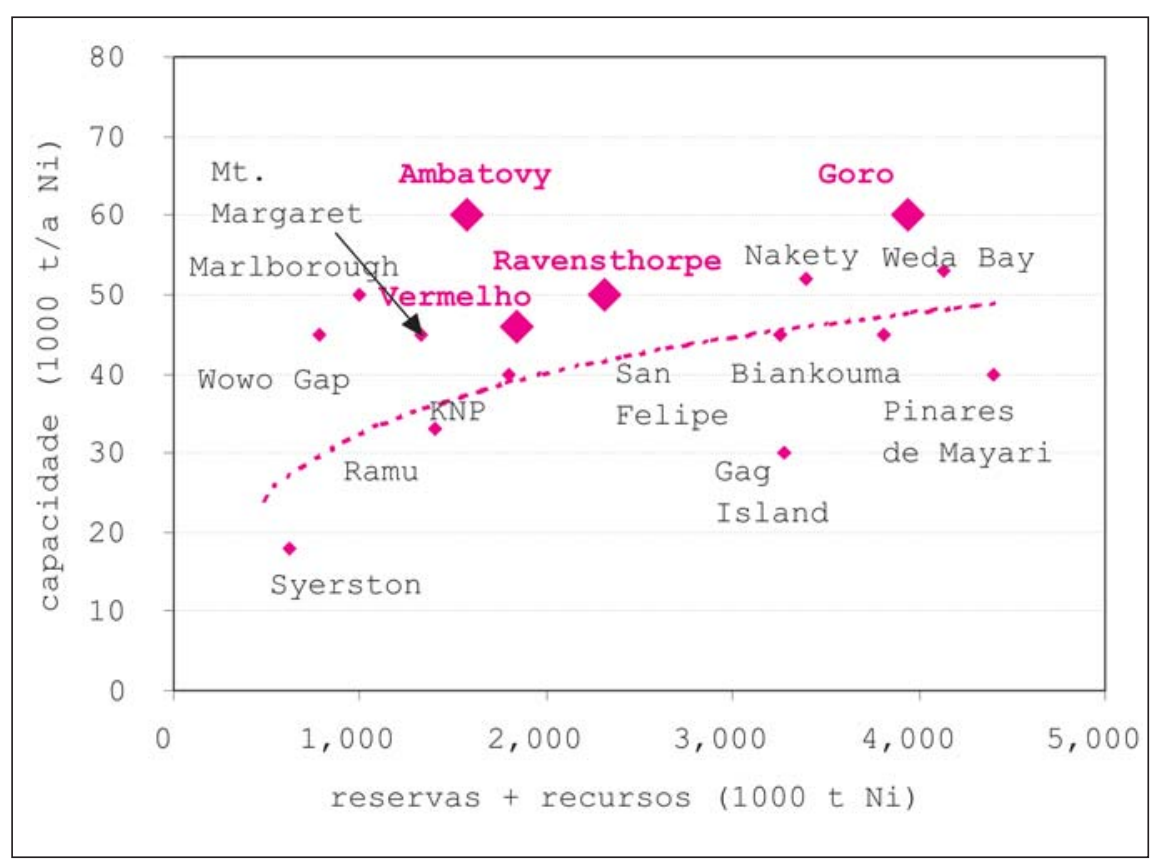

Figura 1 - Capacidade vs. metal contido para os projetos em desenvolvimento e implementação.

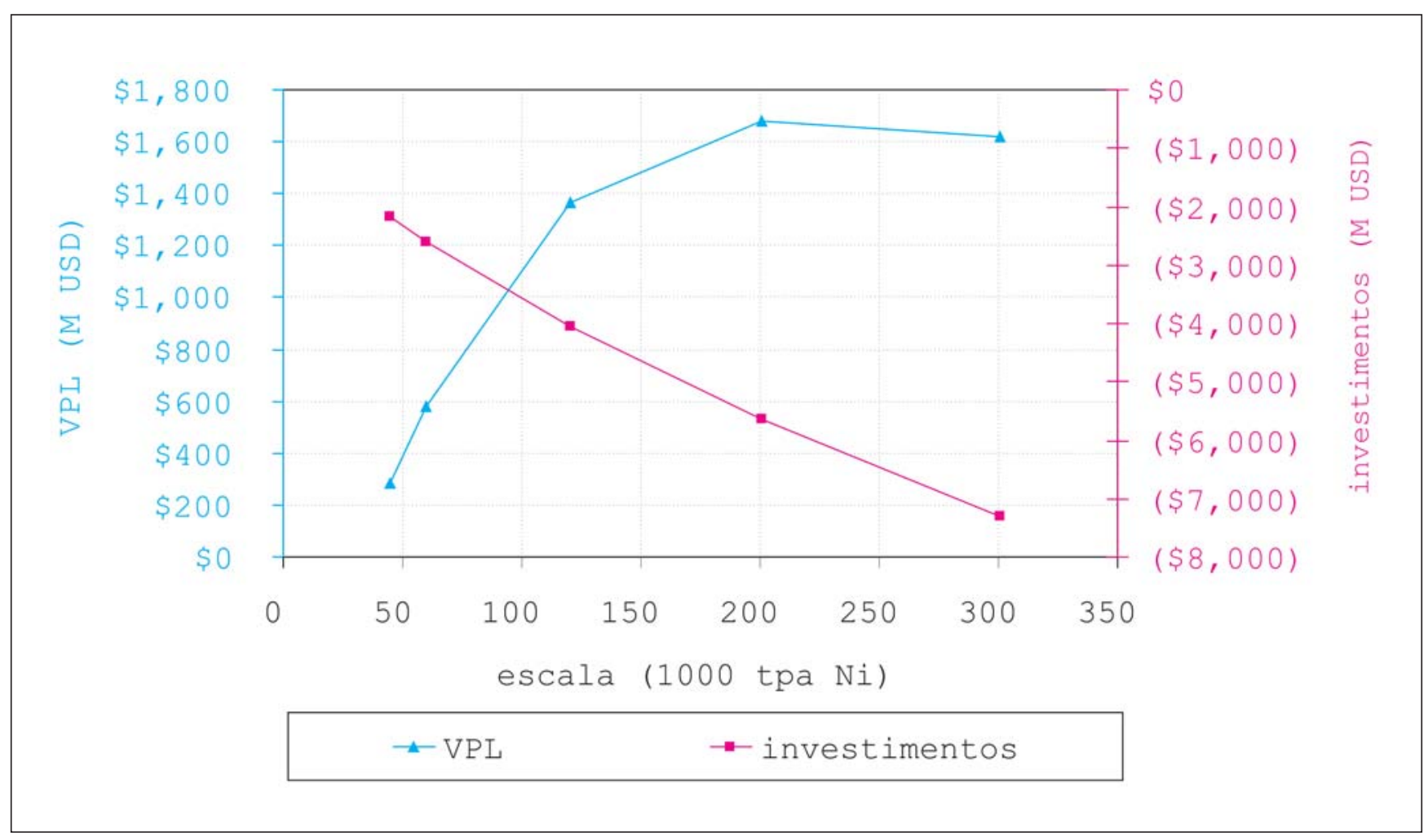

Figura 2 - Valor presente líquido e investimentos vs. escala. 
O valor recomendável a partir da taxa interna de retorno é 200000 t/ano Ni, mas uma análise incremental da taxa interna de retorno mostra que os ganhos entre 120000 t/ano Ni e 200000 t/ano Ni são marginais. A taxa interna de retorno é de $14,6 \%$ para a escala de 45000 t/ano $\mathrm{Ni}$ - um valor no limite de aceitação na indústria de mineração. Para uma taxa de barreira (hurdle rate), mais alta, de $20 \%$ que pode se justificar em condições de maiores riscos -, a escala mínima aceitável seria pouco superior a 100000 t/ano Ni.

A Figura 4 mostra os resultados obtidos para a eficiência de capital e para a taxa interna de retorno nas análises de sensibilidade.

Para uma eficiência de capital maior que 0.3 , a um risco menor que $20 \%$, a menor escala recomendável é 120000 t/ ano Ni. Os riscos de que a eficiência de capital seja menor que 0.3 são de $40 \%$ para a escala de 300000 tpa Ni

Para uma taxa interna de retorno superior a $15 \%$, a um risco máximo de $20 \%$, as escalas recomedáveis são superiores a 120000 t/ano Ni.

\section{Conclusão}

A análise feita na discussão dos resultados do caso-base não é conclusiva, tendo em conta que o valor recomendável para a escala de produção a partir da análise de diferentes indicadores não converge. A análise de sensibilidade permite estabelecer cenários onde os riscos podem ser avaliados e a escala recomendada é de 120000 t/ano Ni.

Considerando, ainda, o porte dos investimentos, o tamanho do mercado e o estágio de maturação da tecnologia de aproveitamento, a escala de produção recomendada para o empreendimento é 120000 t/ano Ni.

Em outros contextos, tais como para o aproveitamento de cobre e ouro, uma abordagem comum para grandes depósitos é delinear reservas e estabelecer os investimentos para a implementação dos projetos, visando a uma vida do empreendimento entre 15 e 20 anos, no máximo - uma escolha que é arbitrária, mas é usual. Adotando-se esses parâmetros para o tamanho de depósito definido, o valor recomendável de escala seria de 90000 t/ano Ni a 120000 t/ano Ni de capacidade, os valores convergem com a conclusão anterior.

\section{Agradecimentos}

Os autores agradecem ao grupo Vale e à Universidade Federal e Ouro Preto pelo apoio durante o desenvolvimento do projeto de pesquisa.

\section{Disclaimer}

Os autor principal é empregado do grupo Vale, que apoiou a pesquisa. Os dados utilizados na pesquisa, no entanto, são todos de fontes públicas e não refletem a opinião ou visão do Grupo sobre o tema de pesquisa.

\section{Referências bibliográficas}

BARBOSA, Fabio. 1Q07 results - May 4, 2007: CVRD. Disponível em: <http:// www.cvrd.com.br/cvrd/media/

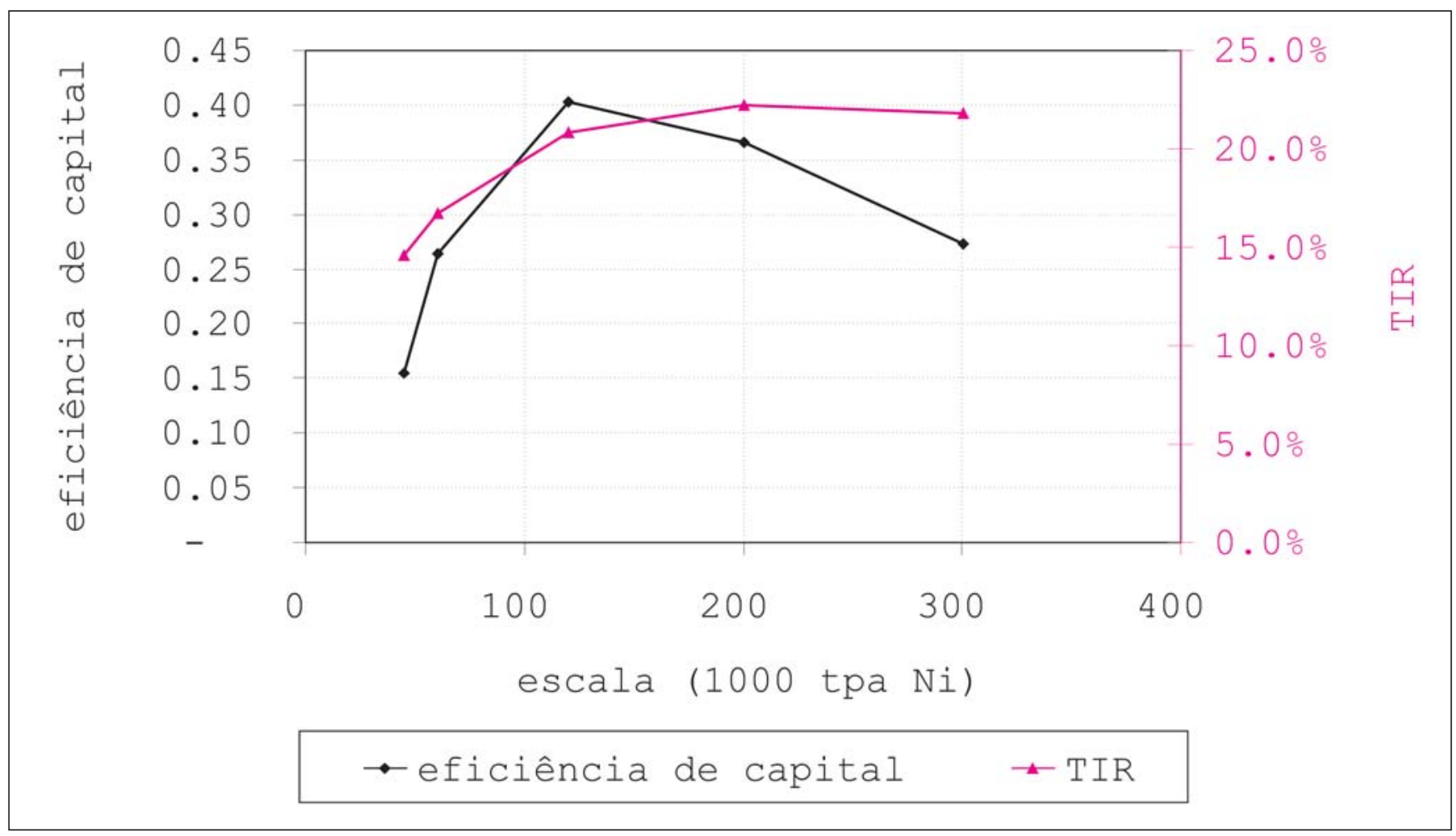

Figura 3 - Eficiência de capital e TIR vs. escala. 


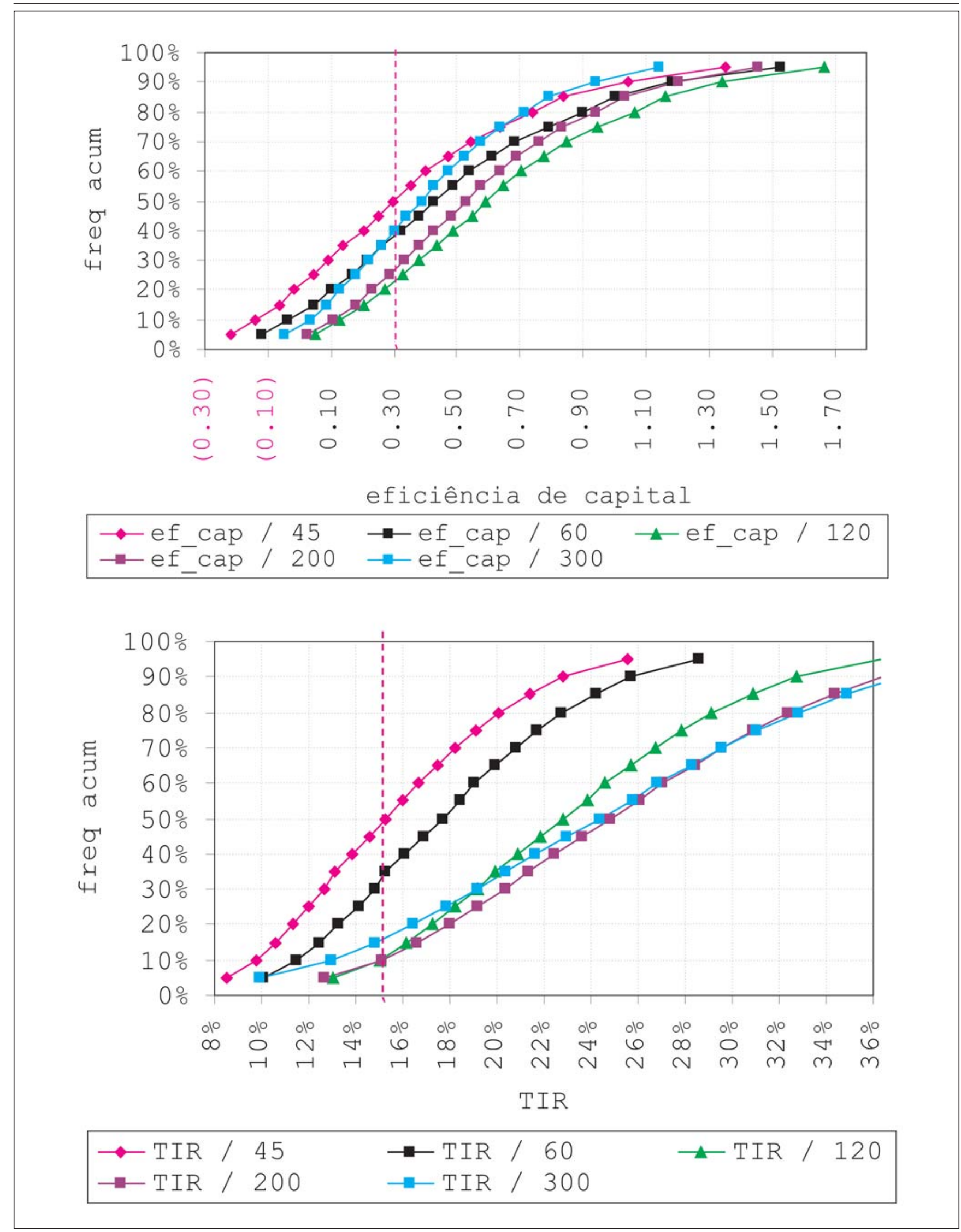

Figura 4 - Eficiência de capital e TIR vs. escala - sensibilidade. 
Avaliação de capacidade de um depósito típico de níquel laterítico aproveitado através de rota...

Cvrdweb1Q07.pdf> Acesso em: 21 Mai. 2007. p. 11.

DALVI, A., BACON, G., OSBORNE, R. The past and the future of nickel laterites. In: PDAC 2004 International convention, 2004, Toronto. Toronto: Prospectors and Developers Association of Canada, 2004. Disponível em: <http://www.pdac.ca/ pdac/publications/papers/2004/ techprgm-dalvi-bacon.pdf $>$ Acesso em: 20 nov. 2006.

DNPM. Informações básicas para o investidor - tributação da mineração no Brasil - análise da situação atual e das mudanças propostas na reforma tributária - Brasília, 2000. Disponível em: h t t p : / / w w w.d n p m.g ov.br/ mostra_arquivo.asp?IDBancoArquivoArquivo=368> Acesso em: 29 mai. 2006.
DOLAN, D., NENDICK, R. Beating USD10 per pound of installed capacity for a laterite nickel plant. In: IMRIE, W. e LANE, D. (eds.). International Laterite Nickel Symposium - 2004. Charlotte, NC, U.S.A, Proceedings. Warrendale, PA: TMS (The Minerals, Metals \& Materials Society), 2004. p. 55-62.

HAND, S. Inco: Inco. Disponível em: <www.inco.com/investorinfo/ p r e s e n t a t i o n s / p d f / BMONesbittFebruary27-06slides.pdf >. Acesso em: 29 mar. 2006.

JOHNSTON, Peter. Minara Presentation February 2006: Minara Resources. Disponível em: <www.minara.com.au/ uploads/ 060227\%20-\%20Minara\% 20presentation \%20Final.pdf $>$. Acesso em 29 mar. 2006. p. 6.
NEUDORF, D., HUGGINS, D. An alternative nickel laterite project development model. In: IMRIE, W. e LANE, D. (eds.). International Laterite Nickel Symposium - 2004, Charlotte, NC, U.S.A, Proceedings. Warrendale, PA, USA: TMS (The Minerals, Metals \& Materials Society), 2004. p. 63-76.

RODRIGUES, R. Definição de capacidade de um depósito médio de níquel laterítico aproveitado através de rota de lixiviação ácida sob pressão. Ouro Preto: Programa de Pós-Graduação em Engenharia Mineral, Universidade Federal de Ouro Preto, 2007. (Dissertação de Mestrado em Engenharia Mineral).

Artigo recebido em 07/03/2008 e aprovado em 20/04/2008.

\section{Descubra as muitas informações da:} Geologia, Mineração,
Metalurgia \& Materiais e Engenharia Civil.
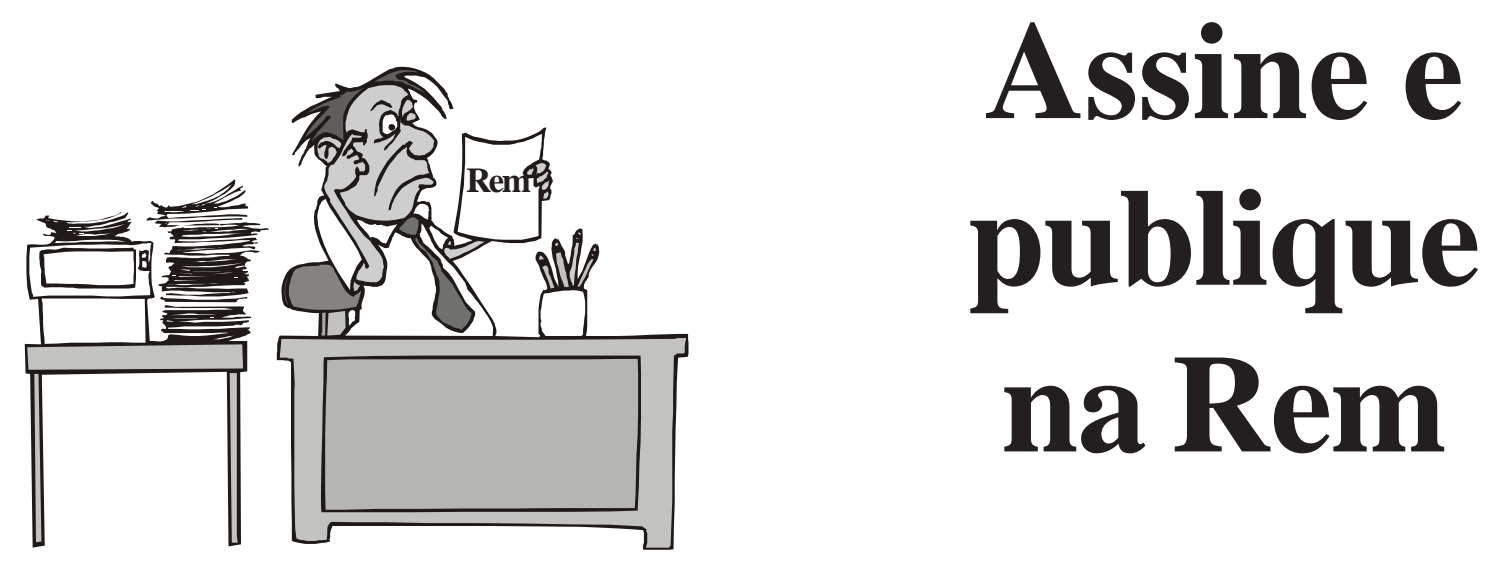

Conheça o nosso novo site: WWW.rem.com.br 